\title{
Clinical characteristics and outcomes of primary versus secondary gastrointestinal mantle cell lymphoma
}

Alessia Castellino ${ }^{1,2}$, Aung M. Tun (1) 1,3, Yucai Wang (1)', Thomas M. Habermann', Rebecca L. King ${ }^{4}$, Kay M. Ristow', James R. Cerhan $\mathbb{1 0}^{5}$, David J. Inwards', Jonas Paludo (1)', Stephen M. Ansell', Thomas E. Witzig ${ }^{1}$ and Grzegorz S. Nowakowski id

\begin{abstract}
Primary gastrointestinal $(\mathrm{Gl})$ mantle cell lymphoma $(\mathrm{MCL})$ is rare and the optimal management is unknown. We reviewed 800 newly diagnosed MCL cases and found 22 primary (2.8\%) and 79 (9.9\%) secondary GI MCL cases. Age, sex, and performance status were similar between primary and secondary cases. Secondary cases had more elevations in lactate dehydrogenase ( $28 \%$ vs $0 \%, P=0.03)$ and a trend for a higher $\mathrm{MCL}$ international prognostic index $(P=0.07)$. Observation or local therapy was more common for primary GI MCL ( $29 \%$ vs $8 \%, P<0.01)$, and autologous stem-cell transplant was more common for secondary GI MCL (35\% vs 14\%, $P<0.05)$. The median follow-up was 85 months. Primary and secondary Gl MCL had similar 5-year progression-free survival (PFS) (30\% vs 28\%, $P=0.59$ ) and overall survival (OS) $(65 \%$ vs $66 \%, P=0.83)$. The extent of $\mathrm{Gl}$ involvement in primary $\mathrm{Gl} \mathrm{MCL}$ affected treatment selection but not outcome, with a 5 -year PFS of $43 \%$ vs $14 \%$ vs $31 \%(P=0.48)$ and OS of $57 \%$ vs $71 \%$ vs $69 \%(P=0.54)$ in cases with single lesion vs multiple lesions in 1 organ vs multiple lesions in $\geq 2$ organs. Less aggressive frontline treatment for primary GI MCL is reasonable. It is unknown whether more aggressive treatment can result in improved outcomes.
\end{abstract}

\section{Highlights}

- Primary GI MCL is rare. These patients tend to be treated less aggressively in the frontline setting yet have similar outcomes as those with secondary GI MCL.

- Less aggressive frontline treatment for primary GI MCL is reasonable given decent outcome, although it is unknown whether more aggressive treatment can result in improved outcomes.

Correspondence: Yucai Wang (Wang.Yucai@mayo.edu) or

Grzegorz S. Nowakowski (Grzegorz@mayo.edu)

'Division of Hematology, Mayo Clinic, Rochester, MN, USA

2Department of Hematology, Santa Croce e Carle Hospital, Cuneo, Italy

Full list of author information is available at the end of the article

These authors contributed equally: Alessia Castellino, Aung M. Tun

\section{Introduction}

Mantle cell lymphoma (MCL) is a distinct subtype of mature B-cell neoplasm constituting approximately $3-10 \%$ of non-Hodgkin Lymphomas ${ }^{1-6}$. It is most commonly diagnosed in older males with the median age of onset approximately 68 years ${ }^{4,5}$. The $t(11 ; 14)(q 13 ; q 32)$ translocation involving $I G H$ and $C C N D 1$ genes is generally considered to be a primary genetic event leading to cyclin D1 overexpression and cell cycle dysregulation ${ }^{7,8}$. Recent studies suggest heterogeneity of MCL with a conventional, more aggressive nodal type and a less common, indolent type with primarily leukemic and

\section{(c) The Author(s) 2021}

(c) (i) Open Access This article is licensed under a Creative Commons Attribution 4.0 International License, which permits use, sharing, adaptation, distribution and reproduction c. in any medium or format, as long as you give appropriate credit to the original author(s) and the source, provide a link to the Creative Commons license, and indicate if changes were made. The images or other third party material in this article are included in the article's Creative Commons license, unless indicated otherwise in a credit line to the material. If material is not included in the article's Creative Commons license and your intended use is not permitted by statutory regulation or exceeds the permitted use, you will need to obtain permission directly from the copyright holder. To view a copy of this license, visit http://creativecommons.org/licenses/by/4.0/. 
splenic involvement ${ }^{9}$. Most patients present with advanced stage disease, and the disease may involve lymph nodes, spleen, as well as extranodal sites ${ }^{7}$. Secondary gastrointestinal (GI) tract involvement in patients with MCL (involving nodal and/or other extranodal tissue) is considered to be common and can be detected at the time of diagnosis and/or relapse. The reported prevalence was $15-30 \%$ in several retrospective studies ${ }^{10-12}$. However, when routine endoscopies were performed in patients with untreated MCL, GI involvement was detected in up to $90 \%$ of patients, although most patients showed no GI symptoms ${ }^{13,14}$. In contrast, primary GI MCL (without additional nodal or extranodal involvement) is a rare presentation that represents only $4-9 \%$ of all primary GI non-Hodgkin lymphomas ${ }^{15,16}$. Primary GI MCL was initially described as multiple lymphomatous polyposis $^{17}$. It can also present as a single polyp, submucosal masses, ulcerative lesions, or diffuse mucosal infiltration $^{13,17-20}$.

As primary GI MCL is uncommon and has significant heterogeneity in clinical presentation, the optimal management remains unclear. In addition, how primary GI MCL compares to secondary GI MCL (i.e., primary nodal MCL with concurrent, biopsy-proven GI involvement at diagnosis) in terms of clinical presentation and treatment outcome has not been well studied. Although a number of case reports are available in the literature, other studies on primary GI MCL are mainly small series with less than 10 cases reporting different outcomes ${ }^{21,22}$. The 5 cases of primary GI MCL with non-blastoid morphology in the British Columbia cohort were observed initially and treated at a later time when indicated, and these patients (presumably with indolent diseases) had a good outcome with a median overall survival (OS) not reached after a follow-up of 4-5 years ${ }^{21}$. In contrast, the 7 patients in another series did poorly overall, with only 1 of 5 patients achieving complete remission after cyclophosphamide, doxorubicin, vincristine, and prednisone (CHOP) chemotherapy while the other 4 had inadequate responses and a median OS of 6 months, although the majority of these patients had blastoid or diffuse variants ${ }^{22}$. An earlier study reported 31 cases of multiple lymphomatous polyposis, but true primary GI MCL cases, as defined per Dawson criteria ${ }^{23}$, were less than 10 in that series ${ }^{17}$. The European MCL Network did the retrospective study of 127 patients with isolated or predominant extranodal MCL that also included 32 patients with primary GI presentation. The median OS of 9.8 years was achieved in the overall study population, but the outcomes of isolated GI cases were not separately reported ${ }^{24}$.

In this study, we sought to compare the clinical characteristics including sites of GI involvement and types of GI lesions, the treatment patterns, and the survival outcomes of patients with primary vs secondary GI MCL, and to investigate whether extent of GI involvement impacted treatment selection and outcome in patients with primary GI MCL.

\section{Methods \\ Patient selections}

The Mayo Clinic institutional review board reviewed and approved the study. Adult patients (age $>18$ ) with biopsy-proven GI involvement of MCL diagnosed between January 1, 1990 and February 28, 2018 were identified from the Mayo Clinic Lymphoma Clinical Database $^{25,26}$. Pathology was reviewed and confirmed by expert pathologists at Mayo Clinic. The Mayo component of the Molecular Epidemiology Resource (MER) of the University of Iowa/Mayo Clinic Lymphoma Specialized Program of Research Excellence (SPORE) ${ }^{27}$, which was established in September 2002, was cross-referenced for additional patients with GI involvement of MCL. Demographic, clinical, and pathological characteristics, treatment information, and follow-up information were abstracted from both databases. Additional chart review was conducted to extract information on GI symptoms, GI involvement pattern, and to verify treatment and follow-up information.

Primary GI MCL was defined as per Dawson criteria $^{23}$ which include (1) absence of palpable superficial lymph nodes; (2) no chest radiographic evidence of enlarged mediastinal lymph nodes; (3) normal total and differential white blood cell (WBC) count; (4) predominant involvement of GI tract with no lymphatic involvement beyond regional lymph nodes; and (5) no evidence of liver and spleen involvement. Patients with elevated WBC count due to neutrophilia but meeting all other Dawson criteria were deemed to have primary GI MCL. The original criterion (4) was based on laparotomy which is not incorporated into routine clinical practice. Therefore, for this study, patients who met Dawson criteria but had obvious non-regional lymph node involvement on imaging (e.g., CT or PET/CT), or biopsy-confirmed bone marrow involvement, were assigned to secondary GI MCL group. Secondary GI MCL was defined as MCL that concurrently involves the GI tract at diagnosis as well as other sites such as non-regional lymph node, liver, spleen, bone marrow, or any other extranodal sites.

Frontline therapeutic strategies were classified into five different categories: (1) observation or localized treatment with surgery or radiation; (2) less aggressive therapy, such as rituximab ( $\mathrm{R}$ )-CHOP, $\mathrm{R}$-bendamustine, $\mathrm{R}$-cladribine, $\mathrm{R}$ monotherapy, and chemotherapy with $\mathrm{CHOP}$ or cladribine, without autologous stem-cell transplant (ASCT); (3) less aggressive therapy followed by ASCT; (4) aggressive therapy, such as with dose-intensified immunochemotherapy with $\mathrm{R}$-maxi-CHOP alternating with rituximab and high-dose cytarabine, or R-CHOP 
alternating with R-DHAP (dexamethasone, cytarabine, and cisplatin), $\mathrm{R}$ plus hyperfractionated cyclophosphamide, vincristine, doxorubicin, and dexamethasone alternating with $\mathrm{R}$ plus high-dose methotrexate and cytarabine (R-HyperCVAD), without ASCT; and (5) aggressive therapy followed by ASCT. Treatment response was evaluated by treating physicians and was abstracted from the databases and/or chart review when necessary.

\section{Statistical analyses}

Baseline clinical and pathological characteristics and therapeutic categories between patients with primary GI MCL and secondary GI MCL were compared using Chisquare or Fisher's exact test. Progression-free survival (PFS) was defined as the time from diagnosis to disease progression or relapse, unplanned retreatment after frontline regimen, or death from any cause. OS was defined as the time from diagnosis to death from any cause. Time to event data (PFS and OS) were analyzed using the Kaplan-Meier method and the Cox proportional hazards model. All statistical analyses were performed using SPSS version 25 (IBM). A $P$ value $<0.05$ was considered statistically significant.

\section{Results}

Prevalence and clinical characteristics of primary GI MCL

A total of 800 patients with MCL were identified from the Mayo Clinic Lymphoma Database and the MER. Twenty-two (2.8\%) patients presented with primary GI MCL while 79 (9.9\%) presented with secondary GI MCL at diagnosis, and all cases had a biopsy-proven histopathologic evidence of GI involvement (Supplemental Fig. 1). Baseline characteristics of primary GI MCL cases are summarized in Tables 1 and 2. The median age at diagnosis was 62 years (range 43-79). There was a substantial male preponderance with a male to female ratio of 19:3. Of the primary GI MCL patients, 95\% (18/19) had an Eastern Cooperative Oncology Group (ECOG) performance status of $<2$. Three (14\%) patients had B symptoms. No patient had an elevated lactate dehydrogenase (LDH). GI symptoms leading to the diagnosis were recorded in $16(73 \%)$ patients, with abdominal pain $(n=7$, $44 \%)$ and GI bleeding $(n=5,31 \%)$ being the most common presentations. The other six (27\%) patients were diagnosed incidentally on routine screening colonoscopy. The GI presentation was heterogeneous and was detected as single lesion (mucosal, polyp, or mass; $n=7,32 \%$ ), multiple lesions confined to 1 organ $(n=7,32 \%)$, and multiple lesions in $\geq 2$ organs $(n=8,36 \%)$. The sites of MCL involvement included colon $(n=15,68 \%)$, small bowel $(n=9,41 \%)$, stomach $(n=7,32 \%)$, rectum $(n=2$, 9\%) and appendix $(n=1,5 \%)$.
Table 1 Baseline characteristics of patients with primary and secondary GI MCL.

\begin{tabular}{|c|c|c|c|c|c|}
\hline & $\begin{array}{l}\text { Primary GI } \\
\mathrm{MCL}, n\end{array}$ & $\%$ & $\begin{array}{l}\text { Secondary GI } \\
\text { MCL, } n\end{array}$ & $\%$ & $P$ value \\
\hline Age & & & & & 0.53 \\
\hline$\leq 60 y$ & 10 & 45.5 & 30 & 38.0 & \\
\hline$>60 y$ & 12 & 54.5 & 49 & 62.0 & \\
\hline Sex & & & & & 0.75 \\
\hline Male & 19 & 86.4 & 66 & 83.5 & \\
\hline Female & 3 & 13.6 & 13 & 16.5 & \\
\hline $\begin{array}{l}\text { ECOG } \\
\text { performance status }\end{array}$ & & & & & $0.68^{\mathrm{a}}$ \\
\hline$<2$ & 18 & 94.7 & 67 & 89.3 & \\
\hline$\geq 2$ & 1 & 5.3 & 8 & 10.7 & \\
\hline Missing & 3 & & 4 & & \\
\hline B symptoms & & & & & $0.76^{\mathrm{a}}$ \\
\hline Yes & 3 & 14.3 & 15 & 19.2 & \\
\hline No & 18 & 85.7 & 63 & 80.8 & \\
\hline Missing & 1 & & 1 & & \\
\hline Sites of Gl involvement & & & & & 0.43 \\
\hline Upper Gl trac & 4 & 18.2 & 7 & 8.9 & \\
\hline Lower Gl tract & 14 & 63.6 & 59 & 74.7 & \\
\hline $\begin{array}{l}\text { Both upper and lower } \\
\text { Gl tract }\end{array}$ & 4 & 18.2 & 13 & 16.5 & \\
\hline Gl lesion type & & & & & 0.12 \\
\hline Single polyp & 3 & 13.6 & 13 & 17.3 & \\
\hline Multiple polyps & 14 & 63.6 & 25 & 33.3 & \\
\hline Mass lesions & 2 & 9.1 & 16 & 21.3 & \\
\hline $\begin{array}{l}\text { Ulceration, erosion, or } \\
\text { nodular lesion }\end{array}$ & 3 & 13.6 & 17 & 22.7 & \\
\hline Random biopsy & 0 & 0 & 4 & 5.3 & \\
\hline Missing & 0 & & 4 & & \\
\hline Histologic subtype & & & & & $1.00^{\mathrm{a}}$ \\
\hline Classic MCL & 21 & 95.5 & 73 & 94.8 & \\
\hline Blastoid variant & 1 & 4.5 & 4 & 5.2 & \\
\hline Missing & 0 & & 2 & & \\
\hline $\mathrm{LDH}$ & & & & & $0.03^{a}$ \\
\hline Normal & 14 & 100.0 & 51 & 71.8 & \\
\hline Elevated & 0 & & 20 & 28.2 & \\
\hline Missing & 8 & 0.0 & 8 & & \\
\hline WBC count & & & & & $0.33^{\mathrm{a}}$ \\
\hline Normal & 12 & 85.7 & 51 & 70.8 & \\
\hline Elevated & 2 & 14.3 & 21 & 29.2 & \\
\hline Missing & 8 & & 7 & & \\
\hline MIPI risk & & & & & 0.07 \\
\hline Low & 10 & 71.4 & 29 & 40.8 & \\
\hline Intermediate & 1 & 7.1 & 25 & 35.2 & \\
\hline High & 3 & 21.4 & 17 & 23.9 & \\
\hline Missing & 8 & & 8 & & \\
\hline
\end{tabular}

${ }^{\mathrm{a}}$ Fisher's exact test. Others were Chi-square test.

Abbreviations: Gl gastrointestinal, MCL mantle cell lymphoma, ECOG PS Eastern Cooperative Oncology Group performance status, $L D H$ lactate dehydrogenase, WBC white blood cell, MIPI MCL international prognostic index.

\section{Clinicopathological characteristics of primary vs secondary GI MCL}

There were no statistically significant differences identified between patients with primary and secondary GI MCL with regard to age ( $\leq 60$ vs $>60)$, sex (male vs 
female), ECOG performance status $(<2$ vs $\geq 2)$, or $\mathrm{B}$ symptoms (Table 1). The distribution patterns of site of GI involvement (upper GI tract vs lower GI tract), GI lesion type, and histology (classic vs blastoid) were similar between patients with primary and secondary GI MCL. Compared with patients with secondary GI MCL, patients with primary GI MCL had fewer elevations in LDH $(P=$ $0.03)$. There was a trend of lower MCL international prognostic index (MIPI) score in patients with primary GI $\operatorname{MCL}(P=0.07)$.

\section{Treatment pattern of primary vs secondary GI MCL}

The treatment patterns for patients with primary and secondary GI MCL are summarized in Table 3. More patients with primary GI MCL were initially observed or treated with localized therapy $(28.6 \%$ vs $7.6 \%, P<0.01)$, while more patients with secondary GI MCL underwent ASCT as part of frontline treatment $(35.4 \%$ vs $13.6 \%, P<$ 0.05).

For patients with primary GI MCL, there was a trend of more aggressive therapy in patients with more extensive GI involvement. Seven patients presented with a single lesion; among those, 2 were initially observed, 2 underwent polypectomy only, and 3 were treated with systemic therapy ( 1 with rituximab alone, 1 with $\mathrm{R}-\mathrm{CHOP}$, and 1 with R-bendamustine). Seven patients presented with multiple lesions in 1 organ; among those, 1 underwent radiotherapy alone, 4 were treated with systemic therapy ( 1 with rituximab alone, 3 with CHOP), 1 was treated with R-bendamustine followed by ASCT, and treatment was unclear for 1 patient. Eight patients presented with multiple lesions involving multiple organs; among those, 1 underwent surgical resection only (multiple polyps in the colon and the rectum), 2 were treated with R-CHOP, 1 was treated with bortezomib-containing regimen, 2 were treated with R-HyperCVAD, and 2 were treated with chemotherapy (1 with $\mathrm{CHOP}$ and 1 with Nordic regimen) followed by ASCT.

\section{Outcomes of primary vs secondary GI MCL}

The median follow-up time for the entire cohort was 85.0 months (95\% CI 53.4-116.6). The median PFS was 32.5 months (95\% CI 21.4-43.6) for patients with primary GI MCL and 38.5 months (95\% CI 22.9-54.2) for patients with secondary GI MCL (Fig. 1a). The 5-year PFS rates were $30.0 \%$ and $28.1 \%$, respectively $(P=0.59)$. The median OS was 94.6 months (95\% CI 56.2-133.0) for patients with primary GI MCL and 136.1 months (95\% CI 39.3-233.0) for patients with secondary GI MCL (Fig. 1b). The 5-year OS rates were $65.3 \%$ and $65.8 \%$, respectively $(P=0.83)$. In the Cox regression model, after adjusting for LDH, patients with primary and secondary GI MCL had similar PFS (HR 1.32, 95\% CI 0.67-2.57, $P=0.42$ ) and OS (HR 0.94, 95\% CI 0.37-2.42, $P=0.90$ ).

\section{Outcomes of primary GI MCL by extent of GI involvement}

In patients with primary GI MCL, the median PFS was 27.8 months (95\% CI 4.8-50.7) for patients with a single lesion, 28.5 months (95\% CI 20.7-36.3) for patients with multiple lesions in 1 organ, and 37.1 months (95\% CI 18.2-56.0) for patients with multiple lesions in $\geq 2$ organs (Fig. 2a). The 5-year PFS rates were 42.9\%, 14.3\%, and $31.3 \%$, respectively $(P=0.48)$. The median OS was 94.6 months (95\% CI 27.8-NA) for patients with a single lesion, not reached (95\% CI 42.7-NA) for patients with multiple lesions in 1 organ, and 224.6 months (95\% CI 19.3-NA) for patients with multiple lesions in $\geq 2$ organs (Fig. 2b). The 5-year OS rates were $57.1 \%, 71.4 \%$, and $68.6 \%$, respectively $(P=0.54)$.

\section{Discussion}

To our knowledge, our study represents one of the largest series of GI MCL that have been reported. We found that primary GI MCL was uncommon (less than 3\% of all MCL), had a heterogeneous clinical presentation, and had similar outcomes compared to secondary GI MCL. Primary GI MCL is considered to be a rare clinical presentation, and it accounted for 4-9\% of all primary GI non-Hodgkin lymphomas ${ }^{15,16}$. The Dawson criteria $^{23}$ of primary GI lymphoma was reported in 1961. There have since been significant advances in imaging and laboratory analysis, namely PET/CT and flow cytometry. Therefore, the diagnosis of primary GI lymphoma in modern practice does not need to follow the Dawson criteria. Practically, a diagnosis of primary GI lymphoma can be made if there is no additional nodal or extranodal involvement of lymphoma in a case of documented GI lymphoma. In the case of primary GI MCL, there can be no nodal, extranodal (including bone marrow), or peripheral blood involvement of MCL. With these criteria, primary GI MCL accounted for $2.8 \%$ of all MCL cases, confirming that this presentation is rare. This result is similar to data reported in the literature. In a previous cohort reported from British Columbia, primary GI MCL accounted for 1.1\% (5/ 440) of the MCL cases $^{21}$. In contrast, secondary GI MCL accounted for approximately $10 \%$ of the MCL cases in our study, somewhat lower than data reported in the literature $(15-30 \%)^{10-12}$, likely due to different study populations, the study era, and different incorporation of endoscopy (i.e., routine endoscopy for staging was not universally done in this cohort).

We found that patients with primary GI MCL cases tended to have lower MIPI scores compared to secondary GI MCL cases. The findings are consistent with a lower disease burden in patients with primary GI MCL. These patients with primary GI MCL were treated less aggressively, with more patients observed or receiving local therapy alone, and fewer patients undergoing ASCT in those treated with systemic therapy. However, the 


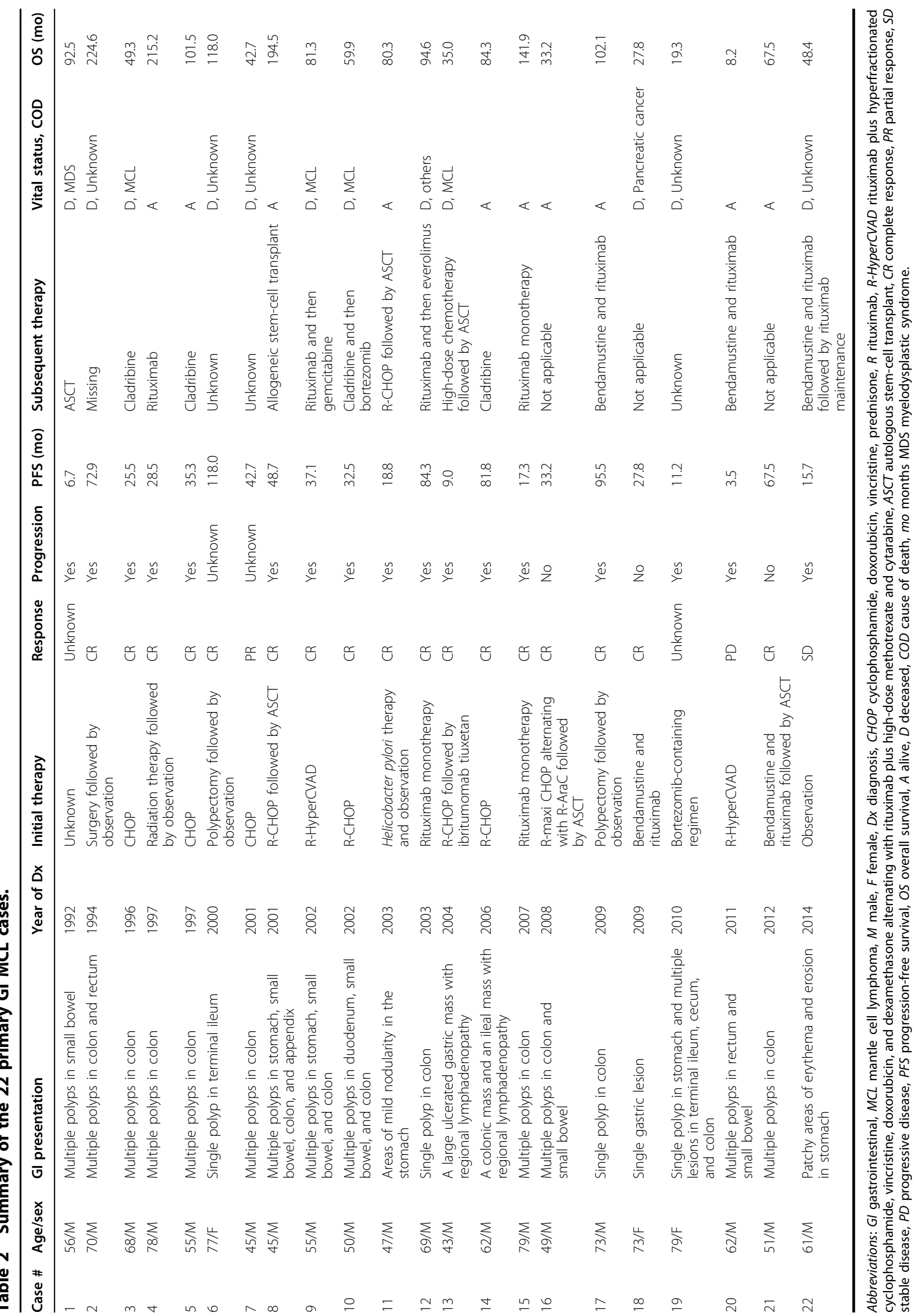


Table 3 Treatment pattern in patients with primary and secondary GI MCL.

\begin{tabular}{|c|c|c|c|c|c|c|c|}
\hline & & \multicolumn{2}{|c|}{ Primary GI MCL } & & \multicolumn{3}{|c|}{ Secondary GI MCL } \\
\hline & & $n$ & \multicolumn{2}{|l|}{$\%$} & \multicolumn{2}{|l|}{$n$} & $\%$ \\
\hline \multicolumn{8}{|l|}{ Frontline therapy } \\
\hline Observation and/or localized therapy & \multicolumn{2}{|r|}{6} & \multicolumn{2}{|l|}{28.6} & \multicolumn{2}{|l|}{6} & 7.6 \\
\hline Less aggressive therapy without ASCT & \multicolumn{2}{|r|}{10} & \multicolumn{2}{|l|}{47.6} & \multicolumn{2}{|l|}{40} & 50.6 \\
\hline Less aggressive therapy with ASCT & \multicolumn{2}{|r|}{2} & \multicolumn{2}{|l|}{9.5} & \multicolumn{2}{|l|}{17} & 21.5 \\
\hline Aggressive therapy without ASCT & \multicolumn{2}{|r|}{2} & \multicolumn{2}{|l|}{9.5} & \multicolumn{2}{|l|}{5} & 6.3 \\
\hline Aggressive therapy with ASCT & \multicolumn{2}{|r|}{1} & \multicolumn{2}{|l|}{4.8} & \multicolumn{2}{|l|}{11} & 13.9 \\
\hline Missing & \multicolumn{2}{|r|}{1} & & & & & \\
\hline \multirow[t]{2}{*}{ Single lesion } & \multicolumn{2}{|c|}{$\begin{array}{l}\text { Multiple lesions in } \\
1 \text { organ }\end{array}$} & \multicolumn{2}{|c|}{$\begin{array}{l}\text { Multiple lesions in } \\
\geq 2 \text { organs }\end{array}$} & \multirow{2}{*}{\multicolumn{2}{|c|}{$n$}} & \multirow[b]{2}{*}{$\%$} \\
\hline & $n$ & $\%$ & $n$ & $\%$ & & & \\
\hline \multicolumn{8}{|l|}{ Frontline therapy } \\
\hline Observation and/or localized therapy & 4 & 57.1 & 1 & 16.7 & & 1 & 12.5 \\
\hline Less aggressive therapy without ASCT & 3 & 42.9 & 4 & 66.7 & & 3 & 37.5 \\
\hline Less aggressive therapy with ASCT & 0 & 0 & 1 & 16.7 & & 1 & 12.5 \\
\hline Aggressive therapy without ASCT & 0 & 0 & 0 & 0 & & 2 & 25.0 \\
\hline Aggressive therapy with ASCT & 0 & 0 & 0 & 0 & & 1 & 12.5 \\
\hline Missing & & & 1 & & & & \\
\hline
\end{tabular}

Abbreviations: Gl gastrointestinal, MCL mantle cell lymphoma, ASCT autologous stem-cell transplant.

treatment outcomes were similar between patients with primary and secondary GI MCL. This suggests that in patients with MCL in the GI tract only, it is reasonable to treat less aggressively, e.g., observation when appropriate, local therapy when feasible, and consider less intensive chemotherapy if systemic therapy is indicated. On the other hand, it is unknown whether more aggressive therapy in patients with primary GI MCL can result in better outcomes. It is not known whether primary and secondary GI MCL have similar biology that dictates the similar outcome. Molecular studies of the GI lymphoma sample in these cases may provide valuable insights.

The extent of GI involvement appeared to have impacted the treatment strategy in our series of patients with primary GI MCL. For instance, patients with a single lesion were more likely to be observed or treated with surgery or radiation alone, while patients with multiple lesions (involving 1 or $2+$ organs) were more likely to receive intensive systemic therapy and ASCT. The treatment outcomes were similar though in patients with different extent of GI involvement. This suggests that in patients with a lower disease burden, less aggressive initial treatment may be reasonable. For example, for patients with a single GI lesion, local therapy, if feasible, may be appropriate. However, we have to note that relapses are still common in patients presenting with one single lesion (as shown in Fig. 2 and detailed in Table 2). Notably, in patients with MCL, GI involvement was found in up to $90 \%$ of the patients when routine endoscopies were done ${ }^{13,14}$. The majority of them were detected by random biopsies of endoscopically normal mucosa ${ }^{13,14}$. Thus, most patients with a single GI lesion may well have multifocal diseases that can result in clinical relapses if no systemic treatment was given initially. In patients with lower burden of GI MCL, e.g., single lesion, while limited therapy at diagnosis and reserving more aggressive systemic treatment for relapse is certainly reasonable and results in decent outcome, it is unknown whether upfront, more aggressive systemic treatment would improve long-term outcome.

The strengths of our study include a systematic review of consecutive MCL cases in the Mayo Clinical Lymphoma Database for identification of GI involvement, a large cohort size of MCL patients, a significant number of primary GI MCL cases for a rare disease, a comprehensive 


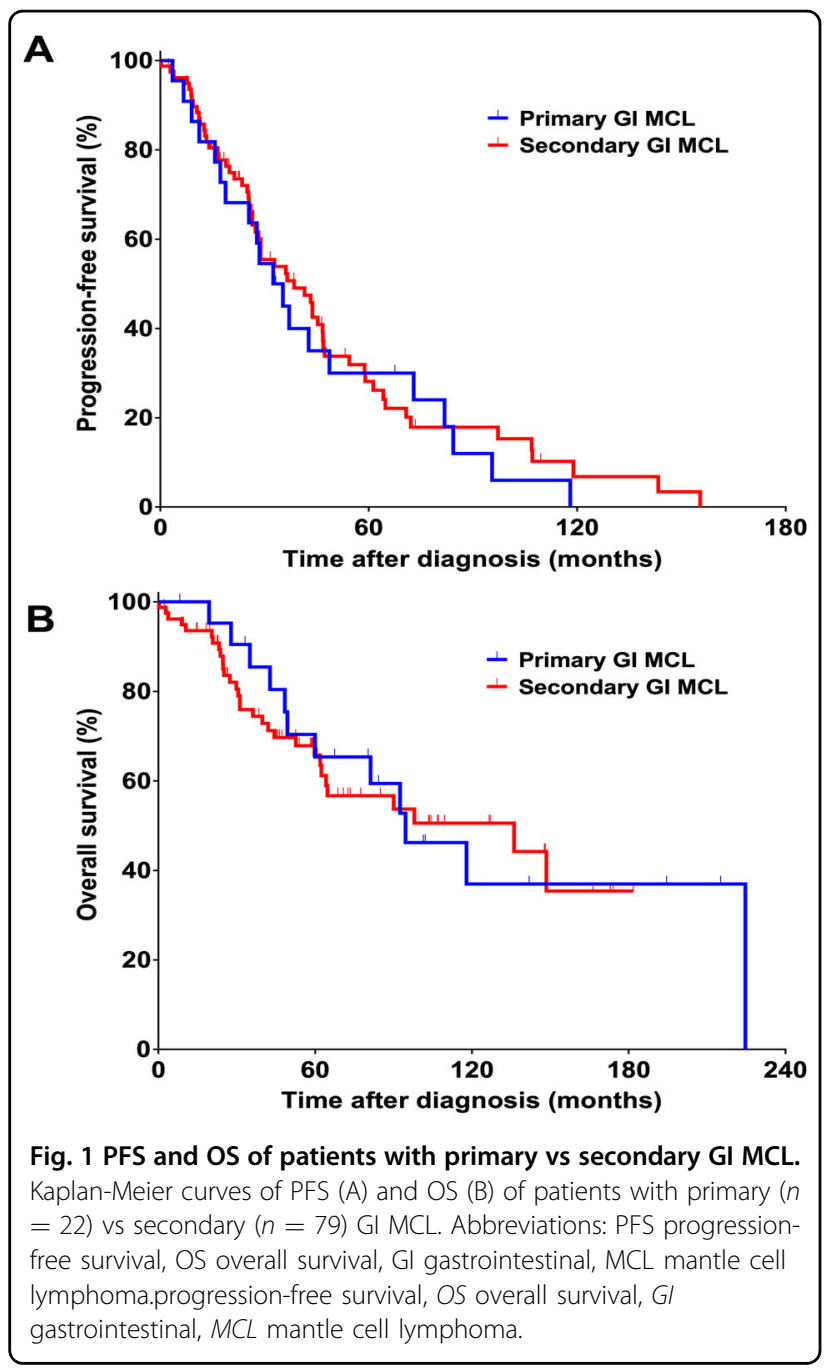

comparison of primary vs secondary GI MCL, as well as closer investigation of the impact of extent of GI involvement which provides potentially important clinical practice implications. The weaknesses of the study include a retrospective design (prospective follow-up only in a subset of patients who were enrolled in MER), a long study period (January 1, 1990-February 28, 2018) that spans different eras of MCL management with potentially different staging and treatment modalities, diverse treatment strategies and regimens over the years, and missing information in some patients who did not follow-up consistently at our institution. Only limited number of patients in this cohort had the opportunity to receive modern treatments such as immunomodulatory drug (IMiD) such as lenalidomide, Bruton's tyrosine kinase (BTK) inhibitors such as ibrutinib, BCL-2 inhibitor such as venetoclax ${ }^{28-35}$. These efficacious novel therapies, as well as the most recently approved CD19 chimeric antigen receptor (CAR) T-cell therapy, may improve the outcomes of both primary and secondary GI MCL ${ }^{36}$.

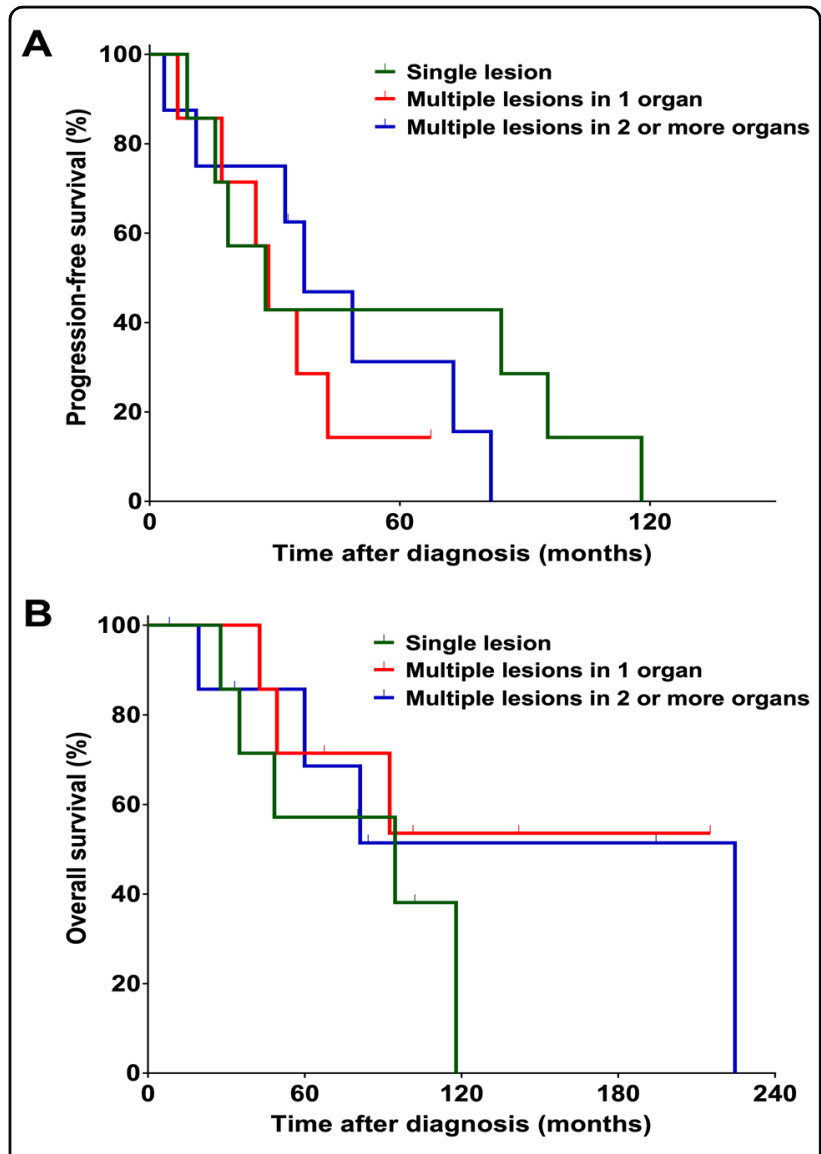

Fig. 2 PFS and OS of patients with primary GI MCL with different extent of GI involvement. Kaplan-Meier curves of PFS (A) and OS (B) of primary GI MCL cases with single lesion $(n=7)$ vs multiple lesions in 1 organ $(n=7)$ vs multiple lesions in $\geq 2$ organs $(n=8)$. Abbreviations: PFS progression-free survival, OS overall survival, Gl gastrointestinal, MCL mantle cell lymphoma-Abbreviations: PFS progression-free survival, OS overall survival Gl gastrointestinal, $\mathrm{MCL}$ mantle cell lymphoma.

In summary, primary GI MCL is a rare and distinct presentation. It tends to be treated less aggressively, especially in patients with a lesser extent of GI involvement, but has a similar outcome to that of secondary GI MCL. Therefore, less aggressive treatment of primary GI MCL is reasonable. Whether an intensified systematic treatment approach for primary GI MCL can result in better outcome is unknown. A better understanding of the molecular biology is needed, and the incorporation of novel therapeutic agents should be explored for patients with primary GI MCL.

\section{Acknowledgements}

Funding provided by the National Cancer Institute, National Institutes of Health (P50 CA097274) and the Predolin Foundation. P50 CA097274 and Predolin Foundation. 


\section{Author details}

'Division of Hematology, Mayo Clinic, Rochester, MN, USA. ${ }^{2}$ Department of Hematology, Santa Croce e Carle Hospital, Cuneo, Italy. ${ }^{3}$ Division of Hematologic Malignancies and Cellular Therapeutics, The University of Kansas, Kansas City, KS, USA. ${ }^{4}$ Division of Hematopathology, Mayo Clinic, Rochester, MN, USA. ${ }^{5}$ Department of Health Sciences Research, Mayo Clinic, Rochester, MN, USA

\section{Conflict of interest}

The authors declare that they have no conflict of interest.

\section{Publisher's note}

Springer Nature remains neutral with regard to jurisdictional claims in published maps and institutional affiliations.

Supplementary Information accompanies this paper at (https://doi.org/ 10.1038/s41408-020-00394-z).

Received: 28 August 2020 Revised: 4 November 2020 Accepted: 24 November 2020

Published online: 07 January 2021

\section{References}

1. Teras, L. R. et al. 2016 US lymphoid malignancy statistics by World Health Organization subtypes. CA Cancer J. Clin. 66, 443-459 (2016).

2. Al-Hamadani, M. et al. Non-Hodgkin lymphoma subtype distribution, geodemographic patterns, and survival in the US: a longitudinal analysis of the National Cancer Data Base from 1998 to 2011. Am. J. Hematol. 90, 790-795 (2015).

3. Shiels, M. S. et al. The epidemic of non-Hodgkin lymphoma in the United States: disentangling the effect of HIV, 1992-2009. Cancer Epidemiol. Biomarkers Prev. 22, 1069-1078 (2013)

4. $\mathrm{Fu}$, S. et al. Trends and variations in mantle cell lymphoma incidence from 1995 to 2013: a comparative study between Texas and National SEER areas. Oncotarget 8, 112516-112529 (2017).

5. Cheah, C. Y., Seymour, J. F. \& Wang, M. L. Mantle cell lymphomaJ. Clin. Oncol. 34, 1256-1269 (2016).

6. Jain, P. \& Wang, M. Mantle cell lymphoma: 2019 update on the diagnosis, pathogenesis, prognostication, and management. Am. J. Hematol. 94 710-725 (2019).

7. Inwards, D. J. \& Witzig, T. E. Initial therapy of mantle cell lymphoma. Ther. Adv. Hematol. 2, 381-392 (2011).

8. $L i$, J. Y. et al. Detection of translocation $t(11 ; 14)(q 13 ; q 32)$ in mantle cell lymphoma by fluorescence in situ hybridization. Am. J. Pathol. 154, 1449-1452 (1999).

9. Swerdlow, S. H. et al. The 2016 revision of the World Health Organization classification of lymphoid neoplasms. Blood 127, 2375-2390 (2016).

10. Samaha, H. et al. Mantle cell lymphoma: a retrospective study of 121 cases. Leukemia 12, 1281-1287 (1998).

11. Chim, C. S. et al. Mantle cell lymphoma in the Chinese: clinicopathological features and treatment outcome. Am. J. Hematol. 59, 295-301 (1998).

12. Yatabe, Y. et al. Significance of cyclin D1 overexpression for the diagnosis of mantle cell lymphoma: a clinicopathologic comparison of cyclin D1-positive MCL and cyclin D1-negative MCL-like B-cell lymphoma. Blood 95, 2253-2261 (2000).

13. Romaguera, J. E. et al. Frequency of gastrointestinal involvement and its clinical significance in mantle cell lymphoma. Cancer 97, 586-591 (2003).
14. Salar, A. et al. Gastrointestinal involvement in mantle cell lymphoma: a prospective clinic, endoscopic, and pathologic study. Am. J. Surg. Pathol. 30, 1274-1280 (2006).

15. Ruskoné-Fourmestraux, A. \& Audouin, J. Primary gastrointestinal tract mantle cell lymphoma as multiple lymphomatous polyposis. Best Pract. Res. Clin. Gastroenterol. 24, 35-42 (2010).

16. Kohno, S. et al. Clinicopathological analysis of 143 primary malignant lymphomas in the small and large intestines based on the new WHO classification. Histopathology 43, 135-143 (2003).

17. Ruskone-Fourmestraux, A. et al. Multiple lymphomatous polyposis of the gastrointestinal tract: prospective clinicopathologic study of 31 cases. Gastroenterology 112, 7-16 (1997).

18. Cornes, J. S. Multiple lymphomatous polyposis of the gastrointestinal tract. Cancer 14, 249-257 (1961).

19. Fraga, M. et al. Mucosal mantle cell (centrocytic) lymphomas. Histopathology 26, 413-422 (1995).

20. Bairey, O., Ruchlemer, R. \& Shpilberg, O. Non-Hodgkin's lymphomas of the colon. Isr. Med. Assoc. J. 8, 832-835 (2006)

21. Abrisqueta, P. et al. Observation as the initial management strategy in patients with mantle cell lymphoma. Ann. Oncol. 28, 2489-2495 (2017).

22. Dasappa, L. et al. Primary gastrointestinal mantle cell lymphoma: a retrospective study. J. Gastrointest. Cancer 45, 481-486 (2014).

23. Dawson, I. M. P., Cornes, J. S. \& Morson, B. C. Primary malignant lymphoid tumours of the intestinal tract. Report of 37 cases with a study of factors influencing prognosis. Br. J. Surg. 49, 80-89 (1961).

24. Morello, L. et al. Mantle cell lymphoma of mucosa-associated lymphoid tissue: a European mantle cell lymphoma network study. HemaSphere 4, e302-e302 (2019).

25. Castellino, A. et al. Role of systemic high-dose methotrexate and combined approaches in the management of vitreoretinal lymphoma: a single center experience 1990-2018. Am. J. Hematol. 94, 291-298 (2019).

26. St-Pierre, F. et al. Detection of extranodal and spleen involvement by FDG-PET imaging predicts adverse survival in untreated follicular lymphoma. Am. J. Hematol. 94, 786-793 (2019).

27. Cerhan, J. R. et al. Cohort profile: the Lymphoma Specialized Program of Research Excellence (SPORE) Molecular Epidemiology Resource (MER) Cohort Study. Int. J. Epidemiol. 46, 1753-1754i (2017).

28. Wang, M. L. et al. Targeting BTK with ibrutinib in relapsed or refractory mantlecell lymphoma. N. Engl. J. Med. 369, 507-516 (2013).

29. Wang, M. et al. Acalabrutinib in relapsed or refractory mantle cell lymphoma (ACE-LY-004): a single-arm, multicentre, phase 2 trial. Lancet 391, 659-667 (2018).

30. Song, Y. et al. Safety and activity of the investigational Bruton tyrosine kinase inhibitor zanubrutinib (BGB-3111) in patients with mantle cell lymphoma from a phase 2 trial. Blood 132, 148 (2018).

31. Tam, C. S. et al. Ibrutinib plus venetoclax for the treatment of mantle-cell lymphoma. N. Engl. J. Med. 378, 1211-1223 (2018).

32. Davids, M. S. et al. Phase I first-in-human study of venetoclax in patients with relapsed or refractory non-Hodgkin lymphoma. J. Clin. Oncol. 35, 826-833 (2017).

33. Eyre, T. A. et al. Efficacy of venetoclax monotherapy in patients with relapsed, refractory mantle cell lymphoma after Bruton tyrosine kinase inhibitor therapy. Haematologica 104, e68-e71 (2019).

34. Tun, A. M. \& Ansell, S. M. Immunotherapy in Hodgkin and non-Hodgkin lymphoma: Innate, adaptive and targeted immunological strategies. Cancer Treat. Rev. 88, 102042 (2020).

35. Goy, A. et al. Phase II multicenter study of single-agent lenalidomide in subjects with mantle cell lymphoma who relapsed or progressed after or were refractory to bortezomib: the MCL-001 "EMERGE" Study. Blood 120, 905 (2012).

36. Wang, M. et al. KTE-X19 CAR T-cell therapy in relapsed or refractory mantle-cell lymphoma. N. Engl. J. Med. 382, 1331-1342 (2020). 